\title{
Novel thoracoscopic lymph node dissection techniques for left upper lobectomy with a fused fissure
}

\author{
Yosuke Matsuura, Junji Ichinose, Masayuki Nakao, Sakae Okumura, Mingyon Mun \\ Department of Thoracic Surgical Oncology, the Cancer Institute Hospital of Japanese Foundation for Cancer Research, Tokyo, Japan \\ Correspondence to: Yosuke Matsuura. Department of Thoracic Surgical Oncology, the Cancer Institute Hospital of Japanese Foundation for Cancer \\ Research, 3-8-31, Ariake, Koto-ku, Tokyo 135-8550, Japan. Email: yosuke.matsuura@jfcr.or.jp.
}

\begin{abstract}
Currently, lobectomy with systematic lymph node dissection (LND) is the standard surgical procedure for early stage, non-small cell lung cancer (NSCLC). Several issues may arise when performing a lobectomy such as a fused fissure, especially during thoracoscopic surgery. In this case, performing hilar LND in a conventional manner may be difficult. Since both hilar and mediastinal LND are essential procedures during surgery for NSCLC, it is crucial to achieve both safe lobectomy and precise systematic LND in fused fissure cases. To address this difficult situation, we have developed the following three novel thoracoscopic techniques that focus on LND: (I) the "anterior fissure first technique", (II) the "\#4L-posterior first technique", and (III) the "anterior \#7 dissection technique". First, the anterior fissure first technique enables the operator to view the entire hilar lymph node, similar to a situation with a complete fissure, and allows the hilar LND to proceed in a conventional manner. Second, when using the \#4L-posterior first technique, the left lower paratracheal (\#4L) LND is straightforward since the left recurrent laryngeal nerve (RLN) has already been dissected, and only the anterior area of \#4L remains to be dissected. Finally, during the anterior \#7 dissection technique, when performing the subcarinal (\#7) LND, we do not dissect between the dorsal side of the trachea and esophagus. Consequently, the bronchial artery (BA) is preserved and postoperative ischemic change in the bronchial stumps is prevented. We believe that our techniques are oncologically appropriate and enable the safe and straightforward execution of precise thoracoscopic lobectomy and systematic LND, even in cases of fused fissures.
\end{abstract}

Keywords: Thoracoscopic surgery; lung cancer; lymph node dissection (LND); fused fissure

Received: 31 March 2020; Accepted: 22 December 2020; Published: 25 May 2021.

doi: 10.21037/ccts-2019-filsc-04-m04

View this article at: http://dx.doi.org/10.21037/ccts-2019-filsc-04-m04

\section{Introduction}

From the 1960s to the present, lobectomy with systematic lymph node dissection (LND) has been the standard surgical procedure for early stage, non-small cell lung cancer (NSCLC) (1). Several difficult situations may arise when performing a lobectomy, such as a fused fissure during thoracoscopic surgery. In general, cases of incomplete fissure may face numerous difficulties when compared to cases of complete fissure, including longer surgical times, greater blood loss, higher rates of postoperative complications including air leakage, and longer duration of chest tube drainage and hospitalization (2). In addition, performing a hilar LND in a conventional manner in the case of an incomplete fissure is may be difficult. Both hilar and mediastinal LND are essential procedures during surgery for NSCLC (3). Therefore, in cases of fused fissure, it is vital to achieve both a safe lobectomy and precise systematic LND. To address this problematic situation, we have developed the following novel thoracoscopic techniques focusing on the LND: (I) "anterior fissure first technique", (II) "\#4L-posterior first technique", and (III) "anterior \#7 dissection technique" $(4,5)$. When using the \#4L-posterior first technique, the left lower paratracheal (\#4L) LND is straightforward since the left recurrent laryngeal nerve (RLN) has already been dissected, and only 


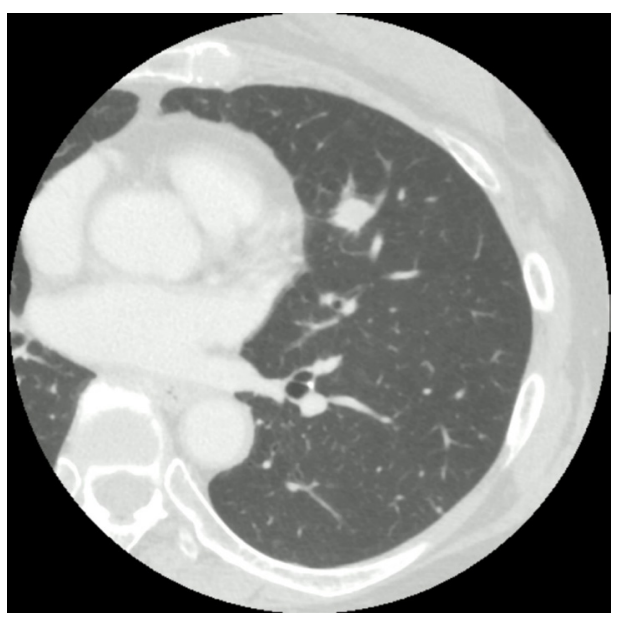

Figure 1 Preoperative chest CT. Thin-slice CT shows a solid nodule in the left $\mathrm{S} 4$ segments. There is no fissure between the upper and lower lobes. CT, computed tomography.

the anterior area of \#4L remains to be dissected. During the anterior \#7 dissection technique, when performing the subcarinal (\#7) LND, we do not dissect between the dorsal side of the trachea and esophagus. Consequently, the bronchial artery (BA) is preserved and postoperative ischemic change in the bronchial stumps is prevented. We believe that our techniques are oncologically appropriate and enable the safe and straightforward performance of precise thoracoscopic lobectomy and systematic LND, even in case of a fused fissure.

\section{Case presentation}

We present the case of a 63 -year-old woman with primary lung squamous cell carcinoma in the left lingular division (Figure 1). The clinical stage was identified as T1bN0M0, IA2 according to the $8^{\text {th }}$ Union for International Cancer Control Tumor Node Metastasis Classification for Lung and Pleural Tumors. We found no fissure on the preoperative chest computed tomography scan (Figure 1). We performed a left upper lobectomy and systematic LND (ND2a-2) via multiple-port thoracoscopic surgery using the following sequence of techniques: (I) \#4L-posterior first technique, (II) anterior fissure first technique, and (III) anterior \#7 dissection technique.

All procedures performed in this study were in accordance with the ethical standards of the institutional and/or national research committee(s), and with the Helsinki Declaration (as revised in 2013). Written informed consent was obtained from the patient for publication of this study and accompanying images. A copy of the written consent is available for review by the editorial office of this journal.

\section{Surgical techniques}

At our institute, we usually perform thoracoscopic lobectomy with systematic LND via four incisions (7, 7, 15, and $30 \mathrm{~mm}$ ) using confronting upside down monitor setting (Figure 2) (6). Two monitors are placed on the patient cranial side, and the monitor display used by the assistants is inverted. The operator stands on the right side of the patient, and the thoracoscopist and secondary assistant stand on the left side in all cases. A $30 \mathrm{~mm}$ utility incision is made in the fifth intercostal space (ICS), and a $7 \mathrm{~mm}$ port for the operator's left hand is inserted in the third ICS. With this arrangement, the operator can freely use both hands and perform a sharp dissection with scissors, electrocautery, or other surgical energy devices. During surgery, the secondary assistant focuses on providing the operator with a better view of the surgical site by placement of two instruments in a $15 \mathrm{~mm}$ incision in the seventh ICS. The thoracoscopist places a $7 \mathrm{~mm}$ port in the fifth ICS to allow visualization of all structures in the chest cavity using a $30^{\circ}$ scope. The thoracoscopist must keep the scope in a horizontal position to ensure that surgical field mirror images do not occur. Each person obtains a correct view of the surgical field when viewing the confronting upside down monitor setting. All procedures are strictly monitor-based, and the role of each port is fixed. We perform LND within the anatomical landmarks, including dissection of the surrounding fatty tissues, according to the European Society of Thoracic Surgeons (ESTS) guideline regarding adequate $\mathrm{LND}$ (7).

\section{Anterior fissure first technique}

The hilar LND is performed in a conventional manner after dividing the fissure.

The anterior fissure first technique consists of the following steps. After incising the mediastinal pleura of the ventral side, we initially identify the anterior basal segmental bronchus $\left(\mathrm{B}^{8}\right)$ located at the cranial side of the inferior pulmonary vein $(\mathrm{PV})$ and identify the interlobar lymph nodes (\#11) (Figure 3A). During this procedure, close attention must be paid to the small branches of the pulmonary artery (PA) and lingular bronchus. The vascular sheath of the anterior basal segmental $\mathrm{PA}\left(\mathrm{A}^{8}\right)$ at the dorsal 


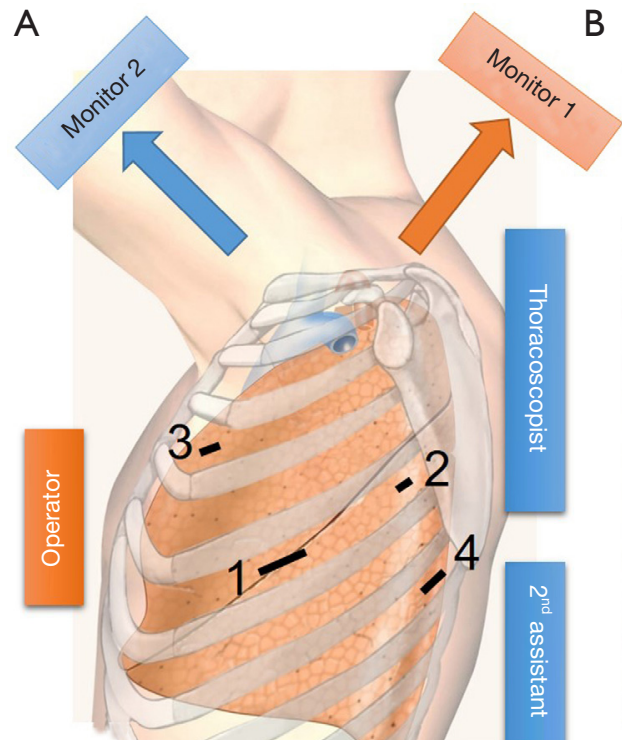

B
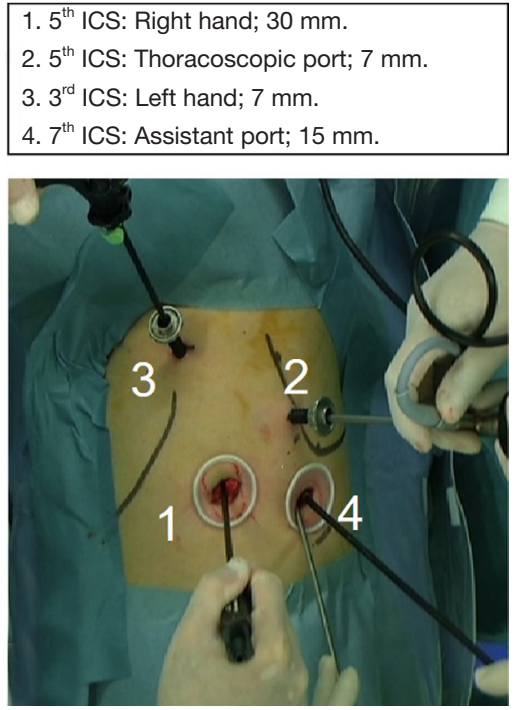

Figure 2 Port placement and confronting upside down monitor setting [(A) schema, (B) surgical view]. Two monitors are placed on the patient cranial side, and the monitor display used by the assistants is inverted (Monitor 2). The operator stands on the right side of the patient and looks at Monitor 1. The thoracoscopist and secondary assistants stand on the left side of the patient and look at Monitor 2. If the thoracoscopist keeps the thoracoscope in a horizontal position, mirror images and disorientation can be avoided. ICS, intercostal space.
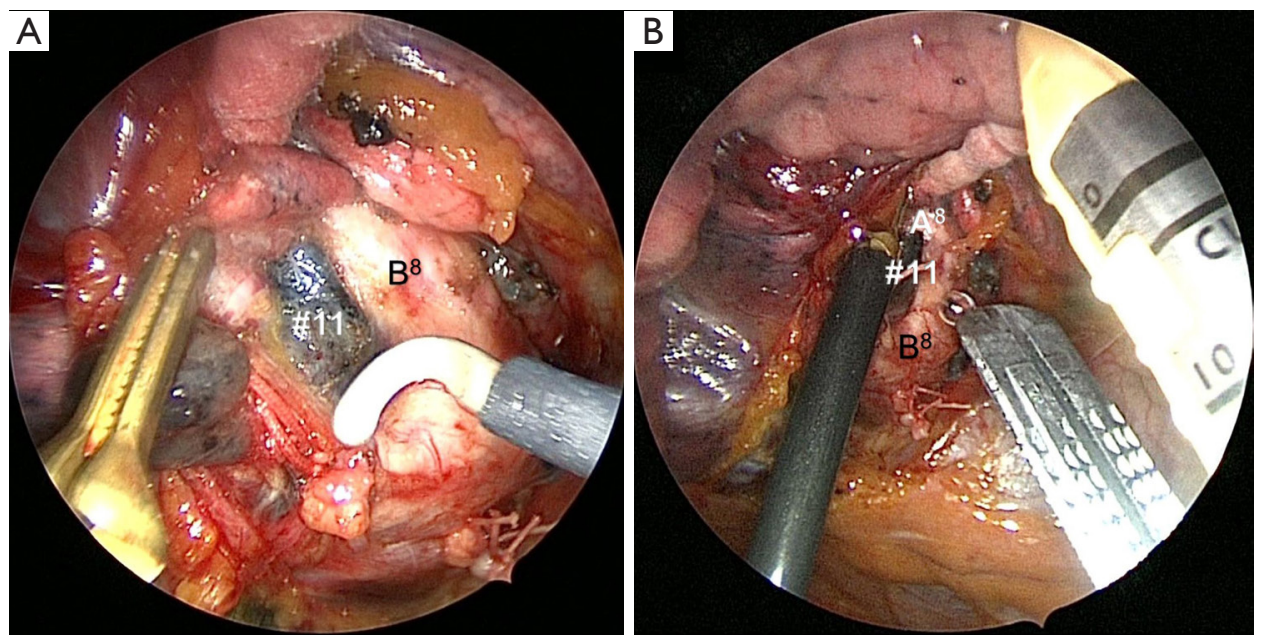

Figure 3 Intraoperative findings of the anterior fissure first technique. Positional relation between the anterior basal segmental bronchus $\left(\mathrm{B}^{8}\right)$ located at the cranial side of the inferior PV and identify the interlobar lymph nodes (\#11) (A). Fused fissures are safely divided by placing the anvil of an automatic articulator (Powered ECHELON FLEX ${ }^{\circledR}$ GTS System, ETHICON, Blue Ash, OH, USA) on the anterior basal segmental $\mathrm{PA}\left(\mathrm{A}^{8}\right)(\mathrm{B})$. PV, pulmonary vein; $\mathrm{PA}$, pulmonary artery.

side of $\mathrm{B}^{8}$ is resected, and the vascular sheath of the $\mathrm{PA}$ is detached towards the hilum. Fused fissures are then safely divided by placing the anvil of an automatic articulator (Powered ECHELON FLEX ${ }^{\circledR}$ GTS System, ETHICON, Blue Ash, OH, USA) on $\mathrm{A}^{8}$ (Figure $3 B$ ). A portion of the fused fissure is often divided using a Harmonic scalpel ${ }^{\circledR}$ (ETHICON, Blue Ash, OH, USA) and electrocautery in conjunction with an automatic articulator. After identification of the superior segmental PA $\left(\mathrm{A}^{6}\right)$, a forceps is passed from the anterior to the posterior, and the fissure 

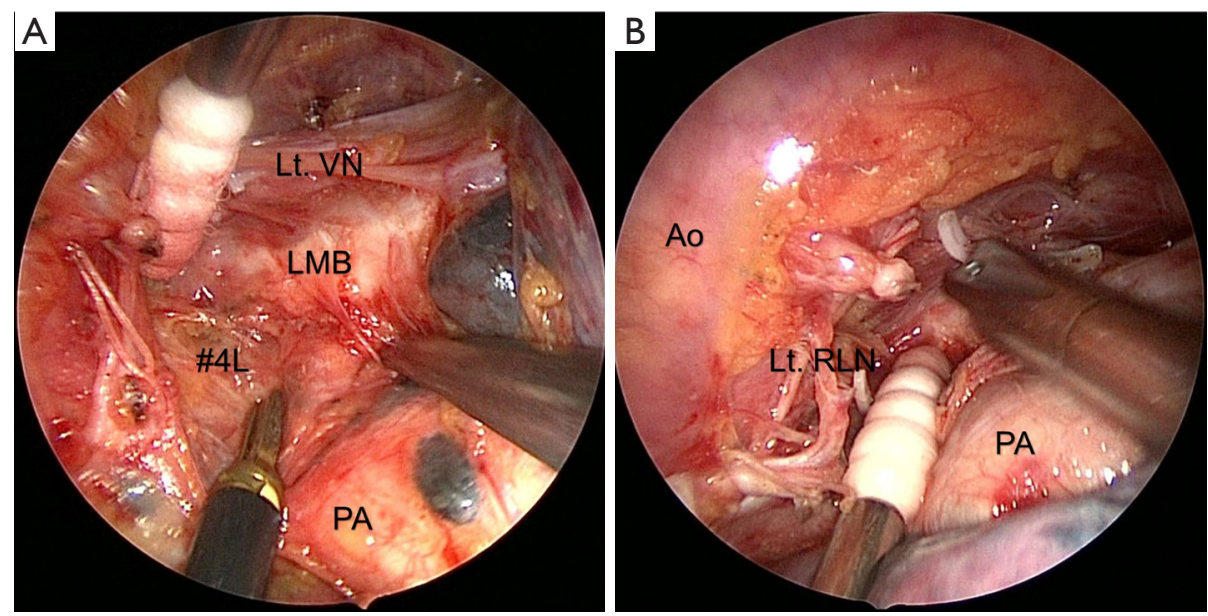

Figure 4 Intraoperative findings after the dissection of the posterior area of the \#4L lymph nodes (A) and after thoracoscopic left upper lobectomy with systematic LND (B). The secondary assistant retracts the lung anteriorly. The retraction applies adequate tension to the left RLN and makes it easier to dissect the left RLN to the distal side. LND, lymph node dissection; RLN, recurrent laryngeal nerve; Lt. VN, left vagus nerve; LMB, left main bronchus; \#4L, left lower paratracheal lymph nodes; PA, pulmonary artery; Ao, aorta; Lt. RLN, left recurrent laryngeal nerve.

division is completed using the automatic articulator. After fissure completion, the hilar LND is performed in a conventional manner. In our conventional method for hilar LND, the BAs are ligated and the hilar lymph nodes are removed en bloc with the BAs.

\section{\#4L-posterior first technique}

The \#4L-posterior first technique involves dissecting the posterior area of the \#4L lymph nodes at the initiation of the surgery. The secondary assistant retracts the lung anteriorly. The mediastinal pleura of the dorsal side is incised just in front of the aorta, and the BAs, directly branching from the aorta, are divided with a surgical energy device. The left main bronchus (LMB) is cranially dissected with scissors. The vagus nerve is exposed, and the left RLN is distally dissected. Dissection of the posterior area of the nodal packet including the \#4L lymph nodes and surrounding structures, such as the LMB, aortic arch, vagus nerve, and left RLN, is completed before approaching the hilar structures (Figure 4A). Subsequently, the lobectomy and mediastinal LND are performed in a conventional manner (Figure 4B). Performing the \#4L LND is straightforward since the left RLN has already been dissected, and only the anterior area of the \#4L remains to be dissected.

\section{Anterior \#7 dissection technique}

The anterior \#7 dissection technique consists of the following steps. After the conventional hilar LND, we lift the LMB and the stump of the left upper PV using a thread and specially modified muscle retractor that may be inserted through a $15 \mathrm{~mm}$ incision. Consequently, the entire subcarinal area may be viewed from the anterior. Subsequently, the \#7 lymph nodes are sequentially dissected from the pericardium and esophagus. The right vagus nerve may be identified, followed by the right main bronchus (RMB). The \#7 lymph nodes are dissected from the RMB and LMB. Finally, the \#7 lymph nodes are clipped and divided from the top of the carina. The preserved BA is clearly recognized from the dorsal side (Figure 5).

\section{Left upper lobectomy with systematic LND}

The complete surgical procedure is described in Video 1.

\section{Discussion}

Our procedure has three important features.

First, we employ an anterior fissure first technique to address a fused fissure during thoracoscopic surgery, which is typically very difficult. Generally, there are two techniques 


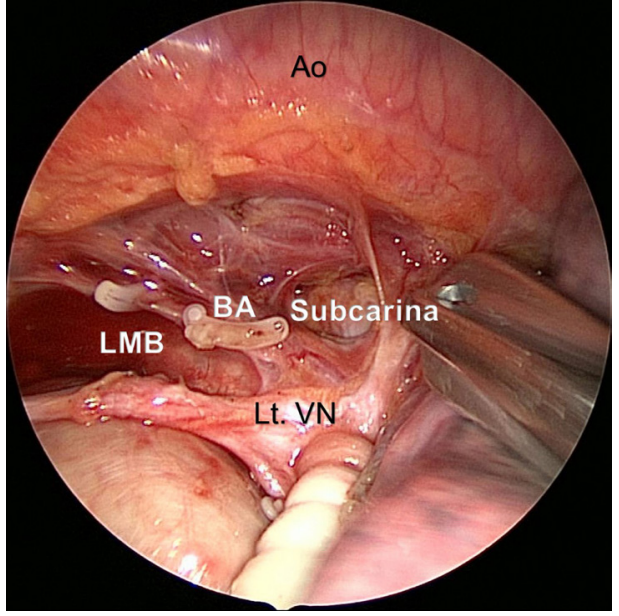

Figure 5 The preserved BA is clearly recognized from the dorsal side. BA, bronchial artery; Ao, aorta; LMB, left main bronchus; Lt. $\mathrm{VN}$, left vagus nerve.

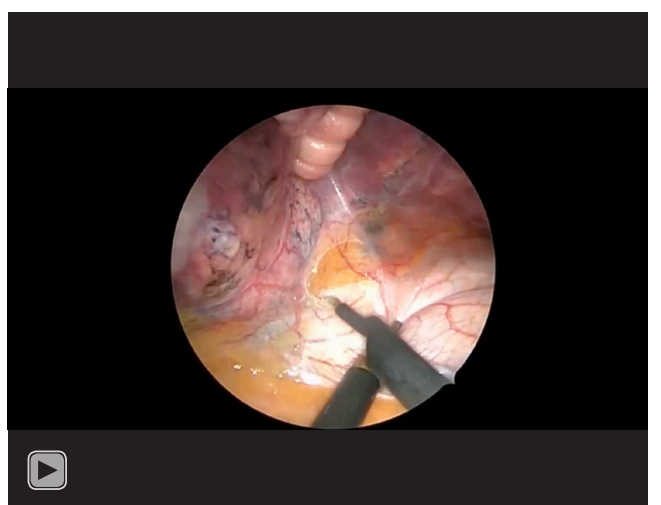

Video 1 Thoracoscopic lymph node dissection techniques for left upper lobectomy with a fused fissure.

used to approach incomplete or fused fissures, either the "hilum first, fissure last" or the "fissure first, hilum last". The hilum first technique, where the hilar structures are dissected before dividing the fissure, is a so-called "fissureless technique", and is considered a useful procedure to reduce postoperative air leakage. In this method, processing may be initiated from the anterior hilar in the following sequence: (I) upper PV, (II) anterior PA $\left(\mathrm{A}^{1+2 a+3}\right)$, (III) apical PA $\left(\mathrm{A}^{1+2 b}\right)$, (IV) left upper bronchus, $(\mathrm{V})$ posterior PA $\left(\mathrm{A}^{1+2 \mathrm{c}}\right)$, (VI) lingular PA $\left(\mathrm{A}^{4+5}\right)$, and (VII) fissure. However, the hilum first technique may be inappropriate for patients with adherent or infiltrating hilar lymph nodes. We do not consider this technique to be oncologically appropriate because it is likely to compromise the hilar lymph nodes. On the other hand, the fissure first technique enables the operator to view the entire hilar lymph node, similar to a situation with a complete fissure, and allows the hilar LND to proceed in a conventional manner. Moreover, a previous report discussion postoperative courses demonstrated that the fissure first technique was feasible and not inferior to the hilum first technique regarding (8). Our group also reported that the thoracoscopic anterior fissure first technique for left lung cancer with an incomplete fissure enabled a hilar LND to be performed in the conventional manner without any increase in the prevalence of air leakage, operating time, or duration of chest tube drainage (5).

Second, we dissected the posterior area of the \#4L lymph node using the \#4L-posterior first technique. Using our procedure, the \#4L LND was straightforward since the left RLN was already dissected and only the anterior area of the \#4L remains for dissection. In a previous study, our group also reported that the \#4L-posterior first approach was a practical procedure which shortened the operation time and reduced blood loss in a thoracoscopic left upper lobectomy with systematic LND (4).

Finally, we reported that the \#7 LND was a significant predictive factor of postoperative ischemic change in the bronchial stumps, which could cause bronchopleural fistulas (9). In addition, reports showed that metastasis to \#7 lymph node concomitant metastasis to the hilar or superior mediastinal nodes was rare in cases of tumors in the right upper lobe and left upper division $(10,11)$. Accordingly, we omitted dissection of the \#7 lymph nodes in cases with left upper division. However, in the case of left lingular division, we dissected the \#7 lymph nodes from the ventral side. During that procedure, we did not dissect between the dorsal side of the trachea and the esophagus in an effort to preserve the BA and prevent bronchopleural fistulas.

We believe that the most important issue for LND is not the removal of individual lymph nodes, but en bloc dissection within the targeted lymph node stations as recommended by ESTS guidelines (7). We also believe that our techniques are oncologically appropriate and enable the safe and straightforward execution of precise thoracoscopic lobectomy and systematic LND, even in cases of used fissures

\section{Acknowledgments}

The authors sincerely thank Dr. Ken Nakagawa for 
instruction in the techniques for performing precise lymph node dissection. The authors also would like to thank Editage (www.editage.com) for English language editing. The video was awarded third prize in the First International Lung Surgery Competition (2019 Masters of Lung Surgery).

Funding: None.

\section{Footnote}

Peer Review File: Available at https://ccts.amegroups.com/ article/view/10.21037/ccts-2019-filsc-04-m04/prf

Conflicts of Interest: All authors have completed the ICMJE uniform disclosure form (available at https://ccts. amegroups.com/article/view/10.21037/ccts-2019-filsc04-m04/coif). The authors have no conflicts of interest to declare.

Ethical Statement: The authors are accountable for all aspects of the work in ensuring that questions related to the accuracy or integrity of any part of the work are appropriately investigated and resolved. All procedures performed in this study were in accordance with the ethical standards of the institutional and/or national research committee(s), and with the Helsinki Declaration (as revised in 2013). Written informed consent was obtained from the patient for publication of this study and accompanying images. A copy of the written consent is available for review by the editorial office of this journal.

Open Access Statement: This is an Open Access article distributed in accordance with the Creative Commons Attribution-NonCommercial-NoDerivs 4.0 International License (CC BY-NC-ND 4.0), which permits the noncommercial replication and distribution of the article with the strict proviso that no changes or edits are made and the original work is properly cited (including links to both the formal publication through the relevant DOI and the license). See: https://creativecommons.org/licenses/by-nc-nd/4.0/.

doi: 10.21037/ccts-2019-filsc-04-m04

Cite this article as: Matsuura Y, Ichinose J, Nakao M, Okumura S, Mun M. Novel thoracoscopic lymph node dissection techniques for left upper lobectomy with a fused fissure. Curr Chall Thorac Surg 2021;3:18.

\section{References}

1. Cahan WG. Radical lobectomy. J Thorac Cardiovasc Surg 1960;39:555-72.

2. Stolz AJ, Schützner J, Lischke R, et al. Predictors of prolonged air leak following pulmonary lobectomy. Eur J Cardiothorac Surg 2005;27:334-6.

3. Asamura H, Chansky K, Crowley J, et al. The International Association for the Study of Lung Cancer Lung Cancer Staging Project: Proposals for the Revision of the N Descriptors in the Forthcoming 8th Edition of the TNM Classification for Lung Cancer. J Thorac Oncol 2015;10:1675-84.

4. Ichinose J, Matsuura Y, Nakao M, et al. A novel procedure of thoracoscopic $4 \mathrm{~L}$ lymph node dissection: $4 \mathrm{~L}$ posterior first technique. J Vis Surg 2020;6:11.

5. Samejima J, Mun M, Matsuura Y, et al. Thoracoscopic anterior 'fissure first' technique for left lung cancer with an incomplete fissure. J Thorac Dis 2016;8:3105-11.

6. Mun M, Ichinose J, Matsuura Y, et al. Video-assisted thoracoscopic surgery lobectomy via confronting upsidedown monitor setting. J Vis Surg 2017;3:129.

7. Lardinois D, Deleyn P, Vanschil P, et al. ESTS guidelines for intraoperative lymph node staging in non-small cell lung cancer. Eur J Cardiothorac Surg. 2006;30:787-92.

8. Decaluwe H, Sokolow Y, Deryck F, et al. Thoracoscopic tunnel technique for anatomical lung resections: a 'fissure first, hilum last' approach with staplers in the fissureless patient. Interact Cardiovasc Thorac Surg 2015;21:2-7.

9. Satoh Y, Okumura S, Nakagawa K, et al. Postoperative ischemic change in bronchial stumps after primary lung cancer resection. Eur J Cardiothorac Surg 2006;30:172-6.

10. Uehara H, Okumura S, Satoh Y, et al. Validity of omission of subcarinal lymph node dissection in patients with cancer of the right upper lobe or left upper division of the lung. JJLC 2008;48:266-72.

11. Okada M, Tsubota N, Yoshimura M, et al. Proposal for reasonable mediastinal lymphadenectomy in bronchogenic carcinomas: role of subcarinal nodes in selective dissection. J Thorac Cardiovasc Surg 1998;116:949-53. 